\title{
Gastrointestinal Bleeding of Obscure Origin Undetected by Multiple Tests for Fecal Occult Blood and Diagnosed Only by Capsule Endoscopy: A Case Report
}

Donald J. Kovacs, MD, and Ted Berk, MD, FACP

The term gastrointestinal bleeding of obscure origin is used to describe bleeding of unknown origin that persists or recurs after a negative initial esophagogastroduodenoscopy and colonoscopy. ${ }^{1}$ We report the case of a middle-aged woman with gastrointestinal bleeding of obscure origin who had 9 stool specimens that tested negative for occult blood but was found to have adenocarcinoma of the distal duodenum on capsule endoscopy. This case illustrates that, in the presence of unexplained iron-deficiency anemia, multiple negative fecal occult blood tests do not exclude the presence of GI blood loss, and that capsule endoscopy is a valuable diagnostic study in this context. ( $\mathrm{J}$ Am Board Fam Med 2006;19:641-2.)

\section{Case Reports}

A 49-year old premenopausal female presented with the sole complaint of fatigue. She had been taking over-the-counter ibuprofen at bedtime. She had perceived no increase in her menstrual blood loss. The only finding on physical examination was slight pallor. Laboratory studies showed iron-deficiency anemia with a hemoglobin of $9.8 \mathrm{~g} / \mathrm{dL}$, hematocrit of $29.6 \%$, serum iron of $9 \mu \mathrm{g} / \mathrm{dL}$, ironbinding capacity of $326 \mu \mathrm{g} / \mathrm{dL}$, and $3 \%$ iron saturation. Three stools tested guaiac negative for blood (Hemoccult II; Beckman Coulter). An esophagogastroduodenoscopy (EGD), colonoscopy, and small bowel follow-through radiograph series were negative. Although 6 additional stools were negative for blood, the patient developed orthostatic dizziness and was found to have a hemoglobin of $6.8 \mathrm{~g} / \mathrm{dL}$. She was transfused with 2 units of packed red blood cells.

The patient was then sent for a capsule endoscopy (at her own expense, because her insurance did not cover it), which revealed a tumor in the proximal small intestine. An EGD with enteroscopy using a pediatric colonoscope revealed adeno-

This article was externally peer reviewed.

Submitted 30 January 2006; revised 2 June 2006; accepted 12 June 2006.

From the Carlisle Regional Medical Center, Carlisle, PA. Conflict of interest: none declared.

Corresponding author: Donald J. Kovacs, MD, 1358 Lutztown Road, Boiling Springs, PA 17007 (E-mail: quakerdoc07@aol.com). carcinoma in the fourth portion of the duodenum. At surgery a large, poorly differentiated tumor was resected, and the patient was found to have metastases in the liver and regional lymph nodes. Less than 1 year from her presentation, the patient died from her cancer.

\section{Discussion}

Two aspects of this case made it remarkable. First, a total of 9 stool specimens tested guaiac negative for blood, even in the face of worsening anemia. Second, routine upper and lower endoscopy failed to reveal the source of bleeding, which was only found using capsule endoscopy.

Although fecal occult-blood testing is recommended as a noninvasive screening method for colorectal cancer, its sensitivity is quite low. A recent study comparing fecal DNA versus fecal occult-blood testing for colorectal cancer screening found the sensitivity of Hemoccult II testing for invasive cancer to be approximately $13 \% .^{2}$ Another study testing 8000 patients with various methods of fecal occult-blood testing showed that the sensitivity of Hemoccult II for the detection of colorectal cancer was $37.1 \%$, compared with $79.4 \%$ with the newer Hemoccult II Sensa. ${ }^{3}$ Guaiac-based tests are even less sensitive for the detection of bleeding from proximal sources because of degradation of hemoglobin as it passes through the GI tract. In our case, gastrointestinal bleeding from the proximal small bowel of sufficient degree to cause severe anemia escaped detection on 9 samples tested for 
occult blood with Hemoccult II. The detection of upper gastrointestinal blood with fecal occult blood testing was studied in healthy volunteers who drank 5,10 , or $20 \mathrm{~mL}$ of their own blood mixed in tomato juice for 3 to 5 days. Consecutive stools were tested from 2 days before until 4 days after the blood ingestion. Only $16 \%$ of the Hemoccult II samples were positive, $64 \%$ of Hemoccult II Sensa samples, and $67 \%$ of HemoQuant samples. ${ }^{4}$

Video capsule endoscopy requires 3 components: a video capsule endoscope approximately the size of a megavitamin pill, a recording device held in a waist belt that can store nearly 60,000 wireless transmitted images from the capsule during an 8-hour period, and a computer workstation for computer-assisted review of the images. The Food and Drug Administration now labels it a first-line test for gastrointestinal bleeding of obscure origin based on its superior diagnostic yield over push enteroscopy, small bowel series, and CT. ${ }^{5}$ Recently, 100 patients with GI bleeding of obscure origin were studied with capsule endoscopy. The sensitivity, specificity, and positive and negative predictive values were $88.9 \%, 95 \%, 97 \%$, and $86.2 \%$, respectively. The presumed causes were discovered in 47 patients; small bowel angiodysplasia and small bowel ulcerations (which are usually because of nonsteroidal anti-inflammatory drugs) accounted for most of the findings. ${ }^{6}$ Additional causes of small bowel bleeding include Crohn's disease, aortoenteric fistulas, diverticula, endometriosis, and hemobilia. ${ }^{1}$ Celiac disease is another disease of the small intestine that commonly presents with iron deficiency. ${ }^{7}$ A meta-analysis of the yield of capsule endoscopy compared with other diagnostic modalities in patients with obscure gastrointestinal bleeding found capsule endoscopy superior to push enteroscopy and small bowel series for diagnosing clinically significant small bowel pathology. ${ }^{8}$ Capsule endoscopy is fairly safe, the primary risk being entrapment of the capsule within the GI tract, which occurs in approximately $0.75 \%$ to $5 \%$ of cases. Such entrapment almost always occurs at the site of the small bowel pathology. Although surgery may be necessary to retrieve the capsule, it would most likely have been required to treat the underlying problem. ${ }^{9}$

Small bowel malignancies are uncommon, accounting for only $2 \%$ of all gastrointestinal neo- plasms, and less than $0.4 \%$ of all cancers in the United States. ${ }^{10}$ However, small bowel tumors are the most frequent cause of obscure gastrointestinal bleeding in patients under 50 years of age. ${ }^{11}$ Adenocarcinomas are the most common type of small bowel tumors and most commonly occur in the duodenum.

\section{Conclusion}

Fecal occult blood testing, even if repeated multiple times, cannot be relied on to exclude gastrointestinal blood loss as the cause of iron deficiency anemia. Capsule endoscopy is extremely useful in the investigation of gastrointestinal bleeding of obscure origin, with a diagnostic yield substantially higher than small bowel follow-through radiograph.

\section{References}

1. AGA technical review: Evaluation and management of occult and obscure gastrointestinal bleeding. Gastroenterology 2000;118:201.

2. Imperiale TF, Ransohoff DF, Itzkowitz SH, et al. Fecal DNA versus fecal occult blood for colorectalcancer screening in an average-risk population. N Engl J Med 2004;351:2704-14.

3. Allison JE, Tekawa IS, Ransom LJ, Adrain AL. A comparison of fecal occult-blood tests for colorectal cancer screening. N Engl J Med 1996;334:155.

4. Rockey DC, Auslander A, Greenberg PD. Detection of upper gastrointestinal blood with fecal occult blood tests. Am J Gastroenterol 1999;94:344-50.

5. Raju GS, Nath SK. Capsule endoscopy. Curr Gastroenterol Rep 2005;7:358-64.

6. Pennazio M, Santucci R, Rondonotti E, et al. Outcome of patients with obscure gastrointestinal bleeding after capsule endoscopy: Report of 100 consecutive cases. Gastroenterology 2004;126:643-53.

7. Marignani M, Angeletti S, Filippi L, et al. Occult and obscure bleeding, iron deficiency anemia, and other gastrointestinal stories (Review). Int $\mathrm{J}$ Mol Med 2005;15:129-35.

8. Triester SL, Leighton JA, Leontiadis GI, et al. A meta-analysis of the yield of capsule endoscopy compared to other diagnostic modalities in patients with obscure gastrointestinal bleeding. Am J Gastroenterol 2005;100:2407-18.

9. Carey EJ, Fleisher DE. Investigation of the small bowel in gastrointestinal bleeding-enteroscopy and capsule endoscopy. Gastroenterol Clin North Am 2005;34:719-34.

10. Jemal A, Murray T, Ward W, et al. Cancer statistics, 2005. CA Cancer J Clin 2005;55:10.

11. Lewis BS, Kornbluth A, Waye JD. Small bowel tumors: yield of enteroscopy. Gut 1991;32:763-5. 